\title{
PERCEPCIONES INFANTILES DE LA ESCUELA EN CONTEXTO DE CONFLICTO ARMADO
}

\section{CHILDREN'S PERCEPTIONS OF SCHOOL IN THE CONTEXT OF ARMED CONFLICT}

\author{
María de Jesús PASALLO ZEPEDA ${ }^{1}$ \\ Ivonne BALDERAS GUTIÉRREZ ${ }^{2}$
}

\begin{tabular}{|lll|}
\hline Recibido & $:$ & 26.02 .2021 \\
Aceptado & $:$ & 17.06 .2021 \\
Publicado & $:$ & 02.08 .2021 \\
\hline
\end{tabular}

\begin{abstract}
RESUMEN: Se abordan las percepciones de niñas y niños que viven en contexto de conflicto armado acerca de la escuela de una comunidad del Estado de Michoacán, México, con quienes se realizaron entrevistas a partir de una investigación acción de mayor alcance, con una postura epistémica crítica, desde una visión no adultocentrista de las infancias. Se parte de nociones de educación y conflicto armado, escuelas en zonas de conflicto armado y niñez, como marco para describir y analizar las voces de las niñas y niños de la institución educativa a partir de dos categorías de análisis, como espacio físico y espacio simbólico. En la primera se expone el apoyo gubernamental. En la segunda la mediación realizada por el docente, y las fortalezas de la escuela que trascienden la función educativa. Se concluye que la escuela se percibe como un lugar seguro para acudir, acompañado del papel del docente como figura que genera confianza.
\end{abstract}

Palabras clave: niñez; educación básica; escuelas; conflicto armado; investigación participativa.

\begin{abstract}
This research addresses the perceptions of children living in the context of armed conflict near the school of a community in the State of Michoacan, Mexico, with whom interviews were conducted based on an action research of greater scope, with a critical epistemic stance, from a nonadult-centered view of childhood. It starts from notions of education and armed conflict, schools in areas of armed conflict and childhood as a framework to describe and analyze the voices of children in the educational institution based on two categories of analysis, such as physical space and symbolic space. The first shows the government support; the second, the mediation carried out by the teacher and the strengths of the school that transcend the educational function. We conclude that the school is perceived as a safe place to go, accompanied by the role of the teacher as a figure that generates confidence.
\end{abstract}

Keywords: childhood; primary education; schools; armed conflict; participatory research.

\footnotetext{
${ }^{1}$ Doctoranda del Programa de Doctorado en Investigación e Innovación Educativa. Benemérita Universidad Autónoma de Puebla - Facultad de Filosofía y Letras. Puebla - México. Email: marypasallo@ gmail.com. ORCID: https://orcid.org/0000-0002-5300-4131

2 Doctora en Educación. Profesora Investigadora en el Doctorado en Investigación e Innovación Educativa. Benemérita Universidad Autónoma de Puebla - Facultad de Filosofía y Letras. Puebla - México. Email: ivonne.balderas@correo.buap.mx. ORCID: https://orcid.org/0000-0002-9633-3269
} 


\section{Journal of the Academy $|144|$}

\section{INTRODUCCIÓN}

El reconocimiento de las voces de niñas y niños acerca de la escuela es fundamental ante la mirada adultocentrista predominante en la atención de las infancias (Unda, 2004). Donde se tiende a desvalorizar el hacer y ser de las niñas y niños, pensándoles como incapaces para generar acciones y participación en sus entornos (Marre, 2014). En esta investigación, la educación, la escuela y todos sus actores, en particular las niñas y niños, se conciben como oportunidades y fortalezas para modificar las situaciones de violencia producidas por conflictos armados.

En tales contextos si la educación se vulnera y violenta, la escuela también lo hace, y con ella sus integrantes, siendo las infancias la población más lastimada. Investigaciones colombianas señalan la forma en que las escuelas en zonas de conflicto han funcionado como campos de formación para la guerra, conformando espacios de reclutamiento, blancos de ataque y de manera general, negando a miles de niñas y niños el derecho a asistir a la escuela (Fajardo, et al., 2018). En tanto, la posibilidad de una educación formal en zonas de conflicto se desquebraja; desaparecen las escuelas, los maestros y los instrumentos pedagógicos, y con ello, se anula el acceso a la educación y a los procesos de aprendizaje y enseñanza (Cervantes, 2014).

En el caso de México se desconocen las repercusiones de los conflictos armados en el ámbito educativo. Se tiene certeza que los conflictos experimentados en varios estados del país han causado efectos devastadores en los sistemas educativos y, por su puesto, en las infancias (Emmerich, 2014). Pero sobre sus peculiaridades apenas se ha comenzado a informar. Tal situación se evidencia en las escasas investigaciones y propuestas en torno al tema, por tanto, el conocimiento producido es mínimo. La Comisión Nacional de los Derechos Humanos [CNDH] (2019) es quien ha realizado un mayor aporte sobre el tema, con el estudio: niñas, niños y adolescentes víctimas de crimen organizado en México, bajo la perspectiva de Derechos Humanos analiza el cumplimiento y ejercicio de estos en las niñas y niños víctimas de la violencia generada por el crimen organizado en México. Se evidencia que el derecho a la educación se ve particularmente afectado, esto debido a la falta de servicios educativos de calidad, altos niveles de ausentismo y deserción escolar. Lo cual, tiene como resultado que se reproduzcan condiciones de pobreza y exclusión social. 


\section{Journal of the Academy $|145|$}

Bajo ese escenario, México figura como uno de los países más violentos y violentados del mundo, violencias relacionadas con el surgimiento del conflicto armado y a los efectos y consecuencias de éste (Instituto para la Economía y la Paz [IEP], 2020). Incluso, es ubicado como un país donde la probabilidad de que una niña o un niño sea asesinado es mucho mayor que en países en guerra tales como Palestina o Siria (Save the Children, 2019). Desde el año 2006 bajo la implementación de la política de seguridad nacional contra las drogas, hasta el día de hoy, no se han logrado reducir las cifras de violencia, cuya tendencia parece acrecentarse (IEP, 2020).

Actualmente se reconocen dos tipos de conflictos, ambos internos, el primero refiere al enfrentamiento entre las fuerzas del Estado, contra los grupos de delincuencia organizada, relacionados con al tráfico de estupefacientes. El segundo, al enfrentamiento de los mismos cárteles entre sí. La mezcla de ambos enfrentamientos ha sido denominada por normas internacionales como conflicto armado, al cumplir con los componentes para ser nombrado así (Organización de las Naciones Unidad [ONU], 2013). Pese a ello, las autoridades no han reconocido la existencia de un conflicto armado, aún con sus múltiples repercusiones. Sin embargo, la aceptación o no del conflicto, no determina los efectos de este, es decir, el conflicto vive y persiste en las comunidades con reconocimiento o no del Estado.

Por otro lado, se plantea un cambio de paradigma en la comprensión y atención de las infancias entendiéndolas como sujetos de derechos, con capacidades, poder de participación y decisión. Donde cada vez se asume que las niñas y los niños muestran procesos más elaborados de autonomía e independencia. Inclusive se ha demostrado cómo tienen mayor control del que tradicionalmente se ha supuesto sobre los asuntos que les afectan a sus cuerpos, mentes y vidas (Marre, 2014). Dependiendo menos que antes de las personas adultas; es sus maneras de explorar el mundo, en la toma de decisiones, como en los procesos de socialización y educación, de ahí que se les entienda como agentes de cambios políticos (Unda 2004).

No obstante, este nuevo paradigma de las infancias no es un derecho que todas las niñas y niños conozcan. Tal es el caso de las infancias que viven en zonas donde hay presencia de conflicto. Usualmente los estudios desarrollados sobre este tema tienden a plantear las necesidades de los menores sin indagar directamente con ellos. Específicamente, en tales contextos, las infancias son las últimas en ser consideradas, como si sus opiniones fueran irrelevantes, 


\section{Journal of the Academy $|146|$}

descontextualizadas o innecesarias. De ahí la importancia de emprender trabajos que visibilicen sus vivencias en esos espacios.

Bajo ese contexto, el objetivo de este trabajo es reconocer las implicaciones educativas desde la mirada y voz de las niñas y los niños que viven, conviven y asisten a la escuela en contexto de conflicto armado. El estudio se realizó en una población con alta presencia de grupos armados, la cual ha sufrido conflictos desde hace más de 11 años. Ubicada al norte del Estado de Michoacán de Ocampo, México. Estado que presenta fenómenos complejos y alarmantes relacionados al conflicto armado interno; homicidios, desapariciones infantiles, migraciones por asilo político, desplazamientos forzados, reclutamiento forzoso, enfrentamientos armados, secuestros y altos índices de orfandad (CNDH, 2020; Pérez et al., 2019; Gobierno de México, 2018; Centro de Justicia para la Paz y el Derecho, 2018; Zepeda, 2016; Fuentes-Díaz, 2015; Comisión Interamericana de Derechos Humanos [CIDH], 2015). Así es como Michoacán se coloca en la quinta posición de los estados más peligrosos de México y en el segundo más violento para las niñas y los niños. Denominado un lugar de alto riesgo para vivir (IEP, 2020).

\section{METODOLOGÍA}

La investigación se planteó bajo el paradigma socio crítico y el enfoque cualitativo, ya que permite comprender a profundidad la realidad desde las subjetividades, significados y contextos de las personas que la integran (Bisquerra, 2012). El método de investigación del estudio más amplio del cual se desprende este texto fue la investigación acción participativa. Lo que se expone corresponde a la fase diagnóstica de la investigación. Para efectos de la investigación, la selección de los participantes se determinó desde los siguientes criterios de inclusión: pertenecientes a la institución educativa rural del estado de Michoacán, México, en contexto de conflicto armado. De acuerdo con el método de investigación acción, la selección de los participantes se dio de forma voluntaria, a través de un proceso interno de análisis y reflexión de participar o decidir no hacerlo. En apego a este planteamiento, se respetó la voluntad y libertad de decisión de las niñas y los niños. Se contó con 16 participantes; nueve niñas de entre los ocho y doce años y siete niños de entre los seis y once años. Todos pertenecientes a la escuela primaria rural. En grados de primer a sexto.

El consentimiento informado se dio a conocer en cuatro niveles: en reunión general de la comunidad; en reunión con los docentes de la escuela; en reunión con madres y padres de 
familia, quienes, por las características del contexto y de la investigación, autorizaron la participación de las niñas y niños a lo largo de la investigación y en las entrevistas. Finalmente, se revisó con las niñas y niños. Asimismo, se realizaron entrevistas semiestructuradas en distintos momentos y tiempos, principalmente al interior de la escuela. El análisis y sistematización de la información para su interpretación, se realizó con el programa ATLAS.ti, con la técnica de análisis cualitativo de contenido (Flick, 2004), a través de matrices descriptivas para la síntesis, saturación de categorías y la interpretación de los ejes de análisis. Inicialmente el análisis fue descriptivo, continuando de forma interpretativa.

\section{RESULTADOS}

Se exponen a partir de la vinculación de la escuela con dos categorías: espacio físico y espacio simbólico. Ambas categorías a partir de la ocupación de las escuelas como espacios que, al contar con una infraestructura, por mínima que sea, es ocupada tanto por el crimen organizado, el ejército y la propia población (Figura 1). Destaca la finalidad de dicha ocupación. En la primera categoría, la ocupación es pragmática, en la segunda, la población recurre a la escuela como entorno de seguridad y protección ante eventos violentos.

\section{Figura 1}

Categorías de las percepciones de la escuela en zonas de conflicto armado

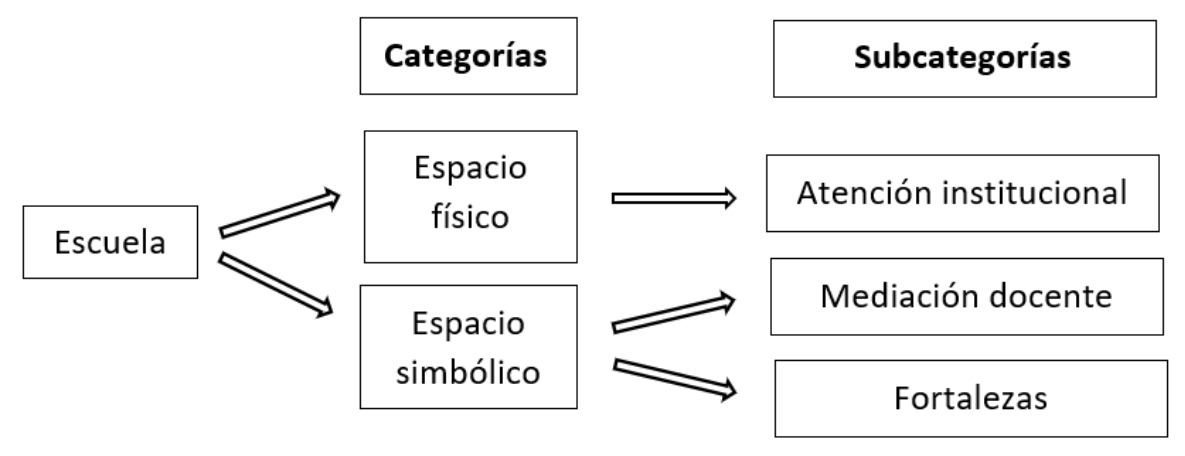

Fuente: Las categorías con base en la ocupación del espacio educativo. Elaboración propia.

\section{Espacio físico}

En diversos estudios se destaca la forma en que las escuelas en los distintos niveles han sido utilizadas por los actores armados como zonas de campamento, bases, refugio o trincheras. Igualmente, para hacer proselitismo, reclutar, almacenar armas, o como lugares de entrenamiento o para realizar acciones de tortura y detención (Ospina-Alvarado, 2018). El 


\section{Journal of the Academy $|148|$}

resultado es que los centros escolares pasan de ser el lugar idóneo para el desarrollo humano de niñas y niños, a ser el lugar de operación del conflicto armado: la escuela como arma y objetivo de guerra.

En este contexto, las niñas y niños mencionan algunos elementos sobre la ocupación de los espacios escolares por parte de los grupos armados, se usan para realizar reuniones, instalarse temporalmente en casas de campaña, hacer uso de algunos servicios que tiene la escuela, como escondite y resguardo de objetos, incluso como escudo durante los enfrentamientos con las fuerzas de seguridad del Estado:

Los armados vienen a la escuela a pedir favores, a que el maestro les saque copias a papeles [...] Hacen juntas en la escuela y citan a la gente aquí [...] Esa camioneta blindada es de los armados, abren la escuela y la meten para esconderla [...] La otra vez, vino el gobierno y los armados se metieron a la escuela con las pistolas y las granadas. Se meten sin avisar y sin pedir permiso (Niño de 11 años, comunicación personal, E6).

En el caso de las fuerzas de seguridad del Estado, la ocupación es con fines militares, al considerarlas como un recurso más del Estado. Ospina-Alvarado (2018) señala que las niñas y niños en esas zonas son violentados por los actores armados ilegales y por los actores legales del Estado:

Cuando han venido los soldados se adueñan del salón y del baño [...] No me gusta que se metan los soldados a la escuela, nos hacen mala cara y no quieren que nos den clases, le dicen al maestro que se vaya a su casa que hoy no de clases (Niño de 6 años, comunicación personal, E1).

La violencia generada por los conflictos armados se instala también en las escuelas. Ello genera afectaciones como ataque y destrucción de las instalaciones escolares. Agrega OspinaAlvarado (2018) que esto es una forma también simbólica de dañar el tejido y la cohesión social. Además, los motivos por los que se atacan a las instituciones escolares son variados. Se considera a las escuelas como símbolos de la autoridad del Estado y, por ende, blancos legítimos de acciones armadas (Fajardo, et al., 2018). Tal destrucción, además del daño al espacio físico, afecta la organización del sistema educativo, las escuelas dejan de funcionar, incluso, algunas no vuelven a abrir sus puertas:

Los armados tumbaron el portón de la escuela y como ya no hay puerta se meten las vacas y se comen las plantas que sembramos con el maestro [...] No hay clases cuando hay enfrentamientos porque los armados no dejan pasar a los maestros. Lo malo es que dejamos de tener clases por muchos días (Niña de 8 años, comunicación personal, E8). 


\section{Journal of the Academy $|149|$}

Mi salón está todo agujerado de las balas y cuando hay lluvia se nos mete el agua. También en las bardas hay agujeros de balas [...] Luego los soldados se meten a la escuela y usan los baños y los descomponen (Niño de 10 años, comunicación personal, E5).

\section{Atención institucional}

La escuela como institución con cierto grado de autonomía y poder, también ha mostrado indiferencia ante las situaciones de conflicto armado. Como señala Márquez (2009) "se ha quedado corta en ese deber ser de la educación frente al conflicto armado" (p. 209). Si se lograra que la escuela deje de ser instrumento para los conflictos armados, se podría favorecer el reconocimiento y la puesta en marcha de mecanismos de autoprotección que hacen posible una mayor cohesión social y una trasformación de los escenarios educativos. Además, es común que el gasto público sea destinado al armamento y al mismo mantenimiento de las fuerzas armadas del Estado, donde los programas educativos quedan debajo de la lista de prioridades.

\section{Espacio simbólico}

Del mismo modo, cuando hay confrontaciones armadas en las comunidades, al ser la escuela el lugar con mayor infraestructura, son utilizadas también como espacio de protección de las comunidades. La ocupación de la escuela significa que el proceso educativo se detiene y que la comunidad educativa se pone en riesgo. Cabría agregar que, en el caso de la ocupación que remite a la comunidad, se vislumbra cómo la escuela, aún y con todas las carencias de seguridad que presenta, sigue siendo un lugar de resguardo y protección que da refugio y seguridad:

La escuela sirve para protegernos, tiene loza y es segura. Cuando vemos helicópteros corremos a la escuela [...] También cuando vienen los soldados y se sabe que va a haber guerra, los papás se meten a la escuela [...] Cuando hay balacera ya no hay clases [...] Es que la escuela es como Dios y afuera es como la guerra (Niña de 10 años, comunicación personal, E15).

Ello trae consigo diversas implicaciones como el abandono permanente de estudiantes y docentes, la interrupción o retroceso en los avances educativos, la escasez de la oferta y la disminución de la calidad de los servicios educativos:

Había un maestro que ya nunca vino y la escuela ya no sirvió por muchos meses y ya cuando mandaron a uno nuevo como que los chiquillos no querían venir de nuevo (Niña de 9 años, comunicación personal, E4). 


\section{Journal of the Academy $|150|$}

A su vez, en comunidades donde la presencia de conflicto armado ha permanecido durante más tiempo y de forma continua, es común que las instituciones educativas incorporen formas de relacionarse que tienden a reproducir las lógicas del conflicto. Añade Fajardo, et al. (2018) que los actores armados suelen imponer en los centros educativos ejercicios autoritarios de poder, que tienen como resultado actos de obediencia, control, desconfianza y miedo situación que disminuye la posibilidad del diálogo, el debate y el encuentro ciudadano. Estas realidades, agrega el mismo autor, suelen transformar la cotidianidad de la escuela, donde se impone la presión, el silencio y la incertidumbre. La escuela entonces tiene que ser protegida para ser un espacio que protege y cuida. De ahí que sea valioso posicionarla primero como centro de la acción comunitaria alrededor de la protección de niñas y niños y segundo como el lugar en donde se aprende sobre los riesgos y los daños que puede generarles a corto y a largo plazo el conflicto armado (Fajardo, et al., 2018).

Así pues, la escuela puede constituirse como un destacado factor protector de las niñas y niños siempre y cuando se constituya en un espacio que vela no solo por la educación formal de la niñez, sino que tome en consideración sus derechos, empoderando y habilitando para ejercerlos y defenderlos. Siendo esto quizás uno de los hallazgos más sorprendentes de los resultados, pues se encontró que los participantes perciben la escuela como un espacio seguro. A pesar de que la escuela sea ocupada y violentada por los conflictos presentados en la comunidad, las niñas y los niños expresan sentirse más seguros al interior de la escuela que en otros espacios que hay en la comunidad:

La escuela es segura, porque adentro de la escuela no tenemos violencia y afuera sí como el otra vez que se metieron los contrarios y comenzó la balacera y amarraron a la gente y con un leño les pegaban, pero nosotros estábamos aquí, todos juntos y el maestro nos cuidó y entre todos vimos qué hacer, porque tenemos más ideas juntos (Niña de 8 años, comunicación personal, E13).

Siendo la escuela un lugar de encuentro con los iguales, de socialización y de generación de relaciones de amistad, en ocasiones son los únicos lazos y las únicas oportunidades de interacción. En las comunidades de conflicto armado, muchos padres no permiten que sus hijas salgan a la calle, para protegerlas, entonces la escuela es la única oportunidad que tienen las niñas para socializar con otros que no sean integrantes de su familia:

Como están los armados en el rancho, mi papá no me deja salir para nada de la casa, ni a mí y a casi ninguna niña que no sea de familia de la maña [...] Solo en la escuela platico con las niñas y ahí juego con ellas, en otro lado no (Niña de 11 años, comunicación personal, E11). 


\section{Mediación docente}

Vinculado a lo anterior, Acosta (2019) menciona que los docentes que trabajan en zonas de conflicto han generado temores que provoca la reacción poco amable hacia las niñas y niños. Mostrando dificultades emocionales y escasa capacidad de interacción con sus alumnos, donde las niñas y niños generalmente les tienen miedo. A su vez se señala que los docentes que trabajan en estas zonas no tienen claridad y preparación para manejar temas relacionados al conflicto. En el caso de este estudio, contrasta con dicho planteamiento ya que las niñas y los niños expresan aprecio y reconocimiento al docente, lo cual representa un importante potencial:

Lo que más me gusta de venir a la escuela es mi maestro, es bueno y nos lleva al río y nos enseña, nos defiende y nos cuenta cuentos, es como un papá [...] Él sí nos platica de lo malo de ser armado. Una vez nos contó cómo surgieron los grupos armados en el rancho (Niño de 6 años, comunicación personal, E17).

\section{Fortalezas}

Este estudio también contrasta con otras investigaciones en la manera en que las niñas y niños que viven en zonas de conflicto armado desvalorizan la escuela, mostrando claras preferencias por trabajar en lugar de asistir a la escuela, incluso, evidencian una mezcla de apatía y terror a estas, evitando acudir. Lo que se contrapone con lo expresado por las niñas y los niños participantes, quienes consideran que la escuela es el mejor espacio de la comunidad para estar, jugar y compartir, en ocasiones mejor que la propia casa, sintiéndose motivados por asistir:

Es bonita la escuela, sirve para trabajar en algo bueno cuando seas grande, yo vengo porque quiero ser doctora y también para hacer amigos y para estudiar cosas que no sé (Niña de 10 años, comunicación personal, E15).

A mí me gusta estar más en la escuela que en mi casa [...] Me pone muy feliz venir a la escuela [...] Es buena y divertida porque aprendemos. También vemos muchos libros y jugamos (Niño de 9 años, comunicación personal, E4).

Incluso, la escuela se percibe como una opción de vida ante la muerte, aspecto trascendente en comunidades en zonas de conflicto con escasas oportunidades para sus habitantes:

Me gusta la escuela, sirve para educar la mente, porque nos hace pensar cosas que no pensamos solos en la casa, aprendemos mucho [...] Es buena para no terminar haciendo trabajos con los armados, ya con estudios se va buscando algo mejor que no te arriesgues y te mueras (Niño de 11 años, comunicación personal, E5).

Como se ha visto hasta ahora, los estudios sobre el tema evidencian las afectaciones; analizar las fortalezas es quizás un reto para la producción de conocimiento en el campo. Dentro de 


\section{Journal of the Academy $|152|$}

estas fortalezas se encuentra la consciencia que tienen los participantes al reconocer que la escuela trasciende la enseñanza de la lectura y la escritura:

La escuela es importante porque aprendes que cuando una persona está pasando por algo malo tienes que ayudarle [...] Aprendemos que los compañeros te deben respetar y tú también respetar, tener principios y valores y no hacer cosas ilegales [...] Aprendes a que no pelees con los demás. Lo más bonito del rancho es la escuela (Niña de 12 años, comunicación personal, E12).

Valdez (2018) señala que las escuelas en zonas de conflicto deberían propiciar y fomentar iniciativas de encuentros democráticos, relaciones de cooperación, de escucha y diálogo. En este caso, las niñas y los niños reflejan que en la escuela surgen prácticas con estas características y que además son disfrutables, con un reconocimiento de los otros como iguales.

\section{CONCLUSIONES}

Ya que el objetivo de este trabajo fue el reconocimiento de las implicaciones educativas en contexto de conflicto armado desde la perspectiva de las niñas y los niños, los resultados son alentadores a partir de la visión de la escuela como espació simbólico desde cuatro aspectos. Primero, se percibe a la escuela como un espacio seguro. Segundo, se aprecia y reconoce el papel del docente. Tercero, a pesar de las condiciones prevalecientes, existe motivación para asistir y estar en la escuela. Finalmente, se destacan otras funciones de la escuela que trascienden la enseñanza de la lectura y escritura.

Como se ha señalado, lo anterior contrasta con los estudios sobre el tema para enriquecerlo. Los resultados de esta investigación también aportan una mirada positiva y alentadora de las potencialidades de la escuela, ya que la mayoría de las investigaciones sobre el tema abordan gran parte de los dolores y las tristezas educativas y es cierto, porque existen y muy grandes. Sin embargo, también es importante visibilizar que hay aspectos rescatables, que sirvan de guía y motivación. Es decir, hay experiencias en donde la educación ha sido una forma no violenta de resistencia.

Pese a ello, el adultocentrismo que marcó tanto épocas antiguas, como modernas, sigue presente en la actualidad, pues las niñas y niños siguen sujetos a los sentidos, sentires, pensamientos y acciones que los adultos de cada época les confieren. En ese tenor se identificó que la mayoría de las investigaciones revisadas centran sus estudios en los adultos que rodean 


\section{Journal of the Academy $|153|$}

a las infancias, trabajando desde ellos y con ellos. En contraste, darles voz a las niñas y niños implica un cambio de paradigma para la construcción de una mirada sobre la infancia de manera contemporánea, abandonando las concepciones históricas de esta. Como plantea Giroux (2003), pensar a la infancia bajo esas categorías es negar los efectos de los problemas reales de las niñas y niños y al mismo tiempo, desviar la atención de los adultos hacia otros temas menos importantes.

De ahí la trascendencia y necesidad de entender que las niñas y los niños son actores sociales y por ende educativos, con voz y experiencias propias que abonan al cambio educativo. Ahora bien, la necesidad de darle voz a las infancias incrementa cuando se abordan y desarrollan temas como los de este trabajo, donde es complejo su abordaje y es ahí donde las perspectivas de las niñas y los niños resultan por demás valiosas, como guías en el camino para lograr un entendimiento más acertado de lo que se debe hacer y cómo realizarlo.

A pesar del gran potencial que tiene la educación, tampoco es capaz de solucionar por sí misma los conflictos y la violencia que generan; sí es una condición para conseguir vivencias diferentes, pues contribuye en la construcción de sociedades más pacíficas, que rechacen la cultura de la violencia y aspiren al fomento de una cultura de paz. De ahí que sea un medio fructífero para emprender acciones en escenarios de conflicto. Lo que se busca es que la educación sea una herramienta de vida y paz y no una herramienta para el conflicto armado. Por lo tanto, tendría que priorizarse el papel de la educación en escenarios de conflicto armado y un elemento para lograrlo es precisamente la escuela. Es necesario entonces plantear estrategias de prevención, atención y resolución de conflictos. Para ello, las instituciones encargadas deben visibilizar en un primer momento, las realidades de las escuelas en zonas de conflicto. A su vez, es necesario la implementación de políticas y estrategias basadas en experiencias internacionales y nacionales para trabajar en pro de una educación de calidad para todas y todos.

Las paredes que protegen a la escuela como una fortaleza, son paredes porosas y minadas que no han podido evitar que el conflicto armado entre hasta el último rincón de la escuela. De ahí que se plantee en este trabajo que el conflicto armado ha traspasado la barrera física y simbólica de la escuela. Pese a ello, la escuela muestra un potencial maravilloso; es una herramienta para vislumbrar vidas distintas, pues es un espacio privilegiado de encuentro con los otros en la construcción de relaciones interpersonales enriquecedoras. Bajo esta mirada, la escuela se 


\section{Journal of the Academy $|154|$}

concibe no solamente como un lugar, sino también como algo vivo que involucra a los sujetos que están dentro de la escuela: niñas, niños y docentes. De ahí que se considere que las infancias y los docentes son semillas fértiles en las escuelas, ellas y ellos como pilar fundamental. Al concebir la escuela como institución de cambio, se deben redoblar esfuerzos para generar metodologías que respondan a las necesidades de los estudiantes, niñas, niños y jóvenes. Las experiencias expuestas en este trabajo sugieren que los esfuerzos en el ámbito educativo deben ser una parte integral de cada sociedad afectada por la violencia.

\section{REFERENCIAS BIBLIOGRÁFICAS}

Acosta, W. A. (2019). Películas de narcos en las aulas. Prácticas alternativas de enseñanza de la historia en Bogotá. Historia y Espacio, 5(53), 221-248. https://doi.org/10.25100/hye.v15i53.8737

Bisquerra, R. (2012). Metodología de la investigación educativa. La Muralla.

Centro de Justicia para la Paz y el Derecho. (2018). Este sexenio tiene los más altos índices de desaparición de niños, niñas y adolescentes. https://cepad.org.mx/2018/04/desaparicionninas-ninos-adolescentes/

Cervantes, D. L. (2014). Consecuencias de los conflictos armados en la educación y sus agentes: una revisión multivocal [Tesis de maestría, Universidad de Granada]. http://digibug.ugr.es/bitstream/handle/10481/32662/TFM_CervantesDuarte.pdf?sequence=1\&is Allowed $=\mathrm{y}$

Comisión Interamericana de Derechos Humanos. (2015). Violencia, niñez y crimen organizado. CIDH. http://www.oas.org/es/cidh/informes/pdfs/violencianinez2016.pdf

Comisión Nacional de los Derechos Humanos (2019). Estudio niñas, niños y adolescentes víctimas de crimen organizado en México. https://www.cndh.org.mx/sites/default/files/documentos/2019-11/Estudio-ninas-ninosadolescentes-victimas-crimen.pdf

Comisión Nacional de Derecho Humanos (2020). Informe Especial Sobre Desplazamiento Forzado Interno en México. http://informe.cndh.org.mx/menu.aspx?id=15008

Emmerich, N. (2014). Estudios sobre el narcotráfico en América Latina: Infancia y narcotráfico en México. Universidad de Belgrano. http://repositorio.ub.edu.ar/handle/123456789/2627

Fajardo, M. M., Ramírez, L. M., Valencia, S. M. y Ospina-Alvarado, M. (2018). Más allá de la victimización de niñas y niños en contextos de conflicto armado: potenciales para la construcción de paz. Universitas Psychologica, 17(1), 1-14. https://doi.org/10.11144/Javeriana.upsy17-1.mavn

Flick, U. (2004). Introducción a la investigación cualitativa. Morata. 
Fuentes-Díaz, A. (2015). Narcotráfico y autodefensa comunitaria en Tierra Caliente, Michoacán, México. Ciencia UAT, 10(1), 68-82. http://www.scielo.org.mx/scielo.php?script=sci arttext\&pid=S2007$\underline{78582015000200068 \& \operatorname{lng}=\mathrm{es} \& \operatorname{tlng}=\mathrm{es}}$

Giroux, H. (2003). La inocencia robada. Juventud, multinacionales y política cultural. Morata. Gobierno de México. (2018). Registro Nacional de Datos de Personas Extraviadas o Desaparecidas. https://datos.gob.mx/busca/dataset/estadistica-de-personas-desaparecidas-nolocalizadas

Instituto para la Economía y la Paz. (2020). Índice de Paz México 2020: Identificar y medir los factores que la impulsan paz. IEP. https://static1.squarespace.com/static/5eaa390ddf0dcb548e9dd5da/t/5eb16bec8506ae2eba951b $\underline{1 \mathrm{~d} / 1588686074573 / \mathrm{ESP}+\mathrm{MPI}+2020+\% 28 \mathrm{web} \% 292 . \mathrm{pdf}}$

Márquez, Q. M. (2009). Enseñanza de la historia del conflicto armado en Colombia: fundamentos para la construcción de propuestas para su enseñanza en el ámbito universitario. Latinoamericana de Estudios Educativos, 5(2), 205-230. https://www.redalyc.org/articulo.oa?id=134116861009

Marre, D. (2014). De infancias, niños y niñas. En V. Llobet (Comp.), Pensar la infancia desde América Latina. Un estado de la cuestión (pp. 9-25). CLACSO.

Organización de Naciones Unidas. (2013). Derecho internacional humanitario. Conceptos básicos. Infracciones en el conflicto armado colombiano. Oficina del Alto Comisionado de las Naciones Unidas para los Derechos Humanos. http://www.oas.org/es/sla/ddi/docs/dih conceptos basicos 2013.pdf

Ospina-Alvarado, M. (2018). Construcción social de niños y niñas: Familias y otros agentes relacionales. CINDE.

Pérez, V. B., Barbosa, M. L., y Castillo, P. M (2019). Entre la invisibilidad y el abandono: un acercamiento cualitativo al desplazamiento interno forzado en México. Comisión Mexicana de Defensa y Promoción de los Derechos Humanos.

Save the Children. (2019). Construyendo una vida mejor con la niñez. https://www.savethechildren.mx/enterate/noticias/construyendo-una-vida-mejor-con-la-ninez

Unda, L. R. (2004). Sociología de la infancia y política social: ¿Compatibilidades posibles? Universitas, Revista de Ciencias Sociales y Humanas, (5), 47-68. http://www.redalyc.org/pdf/4761/476150824003.pdf

Valdez, B. J. (2018). Yo solo quería ser piloto: incorporación de los jóvenes al narcotráfico en Culiacán [Tesis de maestría, Colegio de la Frontera Norte] https://www.colef.mx/posgrado/wp-content/uploads/2018/10/TESIS-Valdez-Batiz-Jairo-Eli.pdf

Zepeda, R. (2016). Violencia en tierra caliente: guerra criminal e intervenciones federales de 2000 a 2014. [Tesis de maestría] El Colegio de México. Centro de Estudios Internacionales. https://repositorio.colmex.mx/concern/theses/s7526c64x?locale=es 